\title{
Colorectal cancer patients exhibit increased levels of galanin in serum and colon tissues
}

\author{
PRZEMYSŁAW KWIATKOWSKI ${ }^{1}$, JANUSZ GODLEWSKI ${ }^{1}$, JACEK KIEŻUN ${ }^{1}$, \\ BARTŁOMIEJ EMIL KRAZIŃSKI ${ }^{1}$ and ZBIGNIEW KMIEĆ ${ }^{1,2}$
}

\author{
${ }^{1}$ Department of Human Histology and Embryology, Faculty of Medical Sciences, University of Warmia and Mazury, \\ 10-082 Olsztyn; ${ }^{2}$ Department of Histology, Medical University of Gdańsk, 80-210 Gdańsk, Poland
}

Received November 2, 2015; Accepted July 26, 2016

DOI: $10.3892 / \mathrm{ol} .2016 .5037$

\begin{abstract}
Galanin (GAL) is a 30-amino acid neuropeptide that is expressed in both the central and peripheral nervous system, including the enteric nervous system (ENS). Increased GAL concentrations have been identified in the blood of colorectal cancer (CRC) patients. The aim of the present study was to assess whether sections of the colon wall containing ENS plexuses or CRC tumor are associated with increased GAL concentrations. Blood samples were collected from $68 \mathrm{CRC}$ patients and 39 healthy volunteers. In addition, samples of CRC tumors and colon wall tissue in close proximity to and distant from the neoplastic tissue were obtained from $22 \mathrm{CRC}$ patients. The GAL concentration of sera and tissue homogenates obtained from three sections of the colon wall (mucosa with submucosa, muscularis externa and CRC tumor) was analyzed by ELISA. The localization of GAL was evaluated using immunohistochemistry and morphometry was used to measure the distribution of GAL-immunoreactive (GAL-Ir) myenteric plexuses in the vicinity of cancer invasion and in sections of the colon wall distant from the tumor. The GAL serum concentration of CRC patients was 2.4 times higher than that of the control group. The GAL concentration was highest in the homogenates of neoplastic tissue and mucosa obtained from the control (distant) section of the intestinal wall, followed by that in the mucosa and muscularis externa proximal to the tumor. The lowest GAL concentrations were identified within the muscular layer of the colon wall located distant from the tumor. Strong GAL immunoreactivity was identified in the cancer cells, intestinal epithelium and the submucosal and myenteric plexuses. Morphometric analysis revealed that the GAL-Ir myenteric plexuses in the vicinity of tumor infiltration were significantly smaller in size than those
\end{abstract}

Correspondence to: Mr. Przemysław Kwiatkowski, Department of Human Histology and Embryology, Faculty of Medical Sciences, University of Warmia and Mazury, 30 Warszawska Street, 10-082 Olsztyn, Poland

E-mail: przemyslaw.kwiatkowski@uwm.edu.pl

Key words: galanin concentration, colorectal cancer, biomarker, serum, myenteric plexus, morphometry, immunohistochemistry in the intact section of the large intestine. Furthermore, no associations were identified between the clinicopathological characteristics of CRC patients and GAL serum and tissue concentration. The increased GAL serum concentrations observed in CRC patients in comparison to healthy controls may result from GAL secretion by CRC tumors, however, other sources of GAL cannot be excluded. The atrophy of myenteric plexuses within close proximity to the tumor may affect the colon function of CRC patients. In conclusion, investigation into the presence of GAL in the colon wall and serum of CRC patients revealed that serum and tissue GAL levels may present useful potential biomarkers in CRC patients.

\section{Introduction}

Colorectal cancer (CRC) is the third most common type of cancer worldwide, accounting for the diagnosis of $\sim 1.4$ million novel cases in 2012 (1). Early diagnosis and prompt treatment substantially improve outcome in CRC patients (2). At present, studies are being conducted in order to identify biological substances that may be involved in cancer development (3). The identification of specific cancer biomarkers is expected to improve early cancer diagnosis in the asymptomatic stage of the disease (4). In humans, galanin (GAL) is a 30-amino acid neuroendocrine peptide, originally isolated from the intestine, which is widely distributed in the central nervous system, endocrine system and autonomic nervous system, including the enteric nervous system (ENS) (5). GAL exhibits various functions, including regulation of feeding and learning, and is involved in responses to nerve injury and pain (5). In the gastrointestinal tract, GAL is involved with intestinal contraction (6). It regulates gastric acid secretion (7) and inhibits the release of pancreatic peptides (8). Notably, increased levels of GAL have been identified within neoplastically-altered tissues of neuroectodermic origin, such as pheochromocytoma (9), pituitary adenoma (10), neuroblastoma (11) and squamous cell carcinoma (12), as well as in malignant melanoma (13). In a previous study, DNA microarray analysis of gene expression in CRC tumors of 51 Korean patients revealed GAL mRNA levels ten times higher than that in normal colon tissues. In addition, GAL expression was observed in colon adenocarcinoma cell lines (LOVO, HCT116, SW480, SW260), whereas GAL mRNA was not detected in the cell lines of lung cancer, 
ovarian carcinoma or testicular carcinoma (14). Kim et al (14) also reported that the CRC patient group exhibited an increased GAL serum concentration when compared with healthy controls, however, the source of the increased GAL serum levels was not determined, and the peptide was not identified in CRC tumors (14).

Due to the fact that colorectal cancer incidence rates for Asian individuals are lower that the rates for Caucasian and African-descended individuals (15), GAL serum concentrations were measured in a cohort of European CRC patients and healthy subjects in the present study. Since our previous study revealed that the expression of neuropeptides (16), including GAL (17), in the ENS ganglia was altered when in close proximity to CRC invasion, the present study additionally aimed to determine the GAL levels in the homogenates of CRC tumors and two dissected sections of the colon wall (mucosa with submucosa and the muscularis externa) both proximal to the tumor and distant from the tumor, which served as the control. Since a reduction in myenteric plexus size and decomposition has been observed in the colon wall of CRC patients (18), the GAL-immunoreactive (GAL-Ir) myenteric plexuses in the vicinity of and distant from CRC invasion in the colon wall were quantitatively assessed.

\section{Materials and methods}

Patients. A total of $68 \mathrm{CRC}$ patients (38 men and 30 women) with a mean age $[ \pm$ standard deviation $(S D)]$ of $68.91 \pm 10.95$ years (range 34-87 years) that underwent surgery at the Warmia and Mazury Oncological Center (Olsztyn, Poland) between October 2012 and November 2013 were included in the present study. Full blood samples were collected preoperatively from all CRC patients and postoperative colorectal samples from resected tissues for the dissection of particular sections of the colon wall were obtained from 22 patients (15 men and 7 women) with a mean age $( \pm$ SD) of $68.79 \pm 10.15$ years (range, 34-87 years). None of the CRC patients suffered from inflammatory bowel disease (IBD) or other gastrointestinal diseases and no patients reported a family history of malignancy. Patients that had undergone neoadjuvant radiotherapy or chemotherapy were excluded from the study.

Blood samples were also obtained from a control group of 39 healthy volunteers ( 9 men and 30 women) with a mean age $( \pm \mathrm{SD})$ of $55.76 \pm 5.47$ years (range, $43-69$ years). The control subjects exhibited no acute or chronic diseases at the time of the study and reported no family history of cancer or IBD. None of the control patients were on medication at the time of study.

The study was approved by the Bioethical Commission of the University of Warmia and Mazury (Olsztyn, Poland) (approval no. 43/2011), and written consent was obtained from all participants.

Collection of serum, tumor samples and colon wall samples. Blood samples were obtained from all patients and healthy volunteers by venipuncture $(\sim 7 \mathrm{ml})$ and centrifuged at $2000 \mathrm{x} \mathrm{g}$ for $10 \mathrm{~min}$ at room temperature. The collected serum (3-4 ml) was then aliquoted and stored at $-20^{\circ} \mathrm{C}$ until further use.

Tissue samples were obtained from 22 CRC patients. Immediately after resection of the cancerous section of the large intestine, two small ( $\sim 1-2 \mathrm{~cm}$ in diameter), full-thickness ( 3-4 mm) intestinal wall samples were collected: one sample was obtained directly from the section of the colon wall adjacent to the tumor and the second sample (control sample) was obtained from the proximal end of the intestine, $\geq 5 \mathrm{~cm}$ from the tumor margin.

The sample of colon wall obtained adjacent from the tumor was carefully dissected into two sections: AM and AMM. The 'AM' section consisted of tumor-adjacent mucosa and submucosa (weight, 0.1-0.2 g) containing submucosal plexuses, whereas the 'AMM' section consisted of tumor-adjacent muscularis externa (weight, 0.1-0.3 g) containing circular and longitudinal muscle layers and the myenteric plexuses localized between them. In addition, a $2 \times 2 \times 2 \mathrm{~mm}$ sample from the periphery of the tumor (weight, 0.1-0.3 g) was also resected. Using the same method, individual sections of the colon were obtained from the non-cancerous section of the colon wall and referred to as the control mucosa and submucosa (DM; distant mucosa and submucosa) and the control muscularis externa (DMM, distant muscularis). The tissue samples intended for the analysis of GAL content in the tissue homogenates were immediately frozen in liquid nitrogen and stored at $-80^{\circ} \mathrm{C}$. Immediately after dissection, the colon wall sections ( 3-4 mm thick) obtained adjacent to or distant from the CRC tumor were fixed in $4 \%$ formaldehyde for $24 \mathrm{~h}$, dehydrated in an ethanol series, embedded in paraffin and cut into $5-\mu \mathrm{m}$ sections.

Measurement of GAL concentration in blood serum, CRC tumor and dissected sections of the colon wall. The concentration of GAL in the sera of 68 patients and 39 healthy volunteers and the tissue homogenates of $22 \mathrm{CRC}$ patients was measured using an ELISA kit for human GAL (\#CEB084Hu; USCN Life Science Inc., Wuhan, China). Cryopreserved tissues were thawed and rinsed in ice-cold phosphate-buffered saline (PBS; pH 7.4) and weighed. Following separation by the use of surgical scalpel and tweezers, $100 \mathrm{mg}$ tissue was homogenized (Ultra-Turrax T10 homogenizer; IKA -Werke $\mathrm{GmbH} \&$ Co. KG, Staufen, Germany) in $5 \mathrm{ml}$ PBS on ice three times for $20 \mathrm{sec}$. The homogenates were then centrifuged at $5,000 \mathrm{x} \mathrm{g}$ for $5 \mathrm{~min}$ at $4^{\circ} \mathrm{C}$ and the supernatants were stored at $-80^{\circ} \mathrm{C}$ for further processing. All analysis was performed in duplicate according to the manufacturer's instructions. The optical density was measured using a microplate reader (Infinite M200 Pro; Tecan Group Ltd., Männedorf, Switzerland). Standard curves were generated for GAL concentrations of $1000.00,333.33,111.11,37.04$ and $12.35 \mathrm{pg} / \mathrm{ml}$. The intra- and inter-assay coefficients of variation for the GAL assays were $<12 \%$. The data were linearized by plotting the log of GAL concentration vs. the log of the optical density, and the results were expressed in terms of pg of GAL per $\mathrm{ml}$ of serum and as pg of GAL per $g$ of tissue.

Immunohistochemistry (IHC). IHC, defined as positive immunoreactivity for GAL, was performed as described previously by Godlewski et al (19) with certain modifications. The sections were subjected to antigen retrieval by microwaving for $20 \mathrm{~min}$ in retrieval solution buffer ( $\mathrm{pH}$ 6.0; Leica, Wetzlar, Germany) and incubated with $3 \% \mathrm{H}_{2} \mathrm{O}_{2}$ in methanol for $10 \mathrm{~min}$ followed by $2.5 \%$ normal horse serum (Vector Laboratories, 

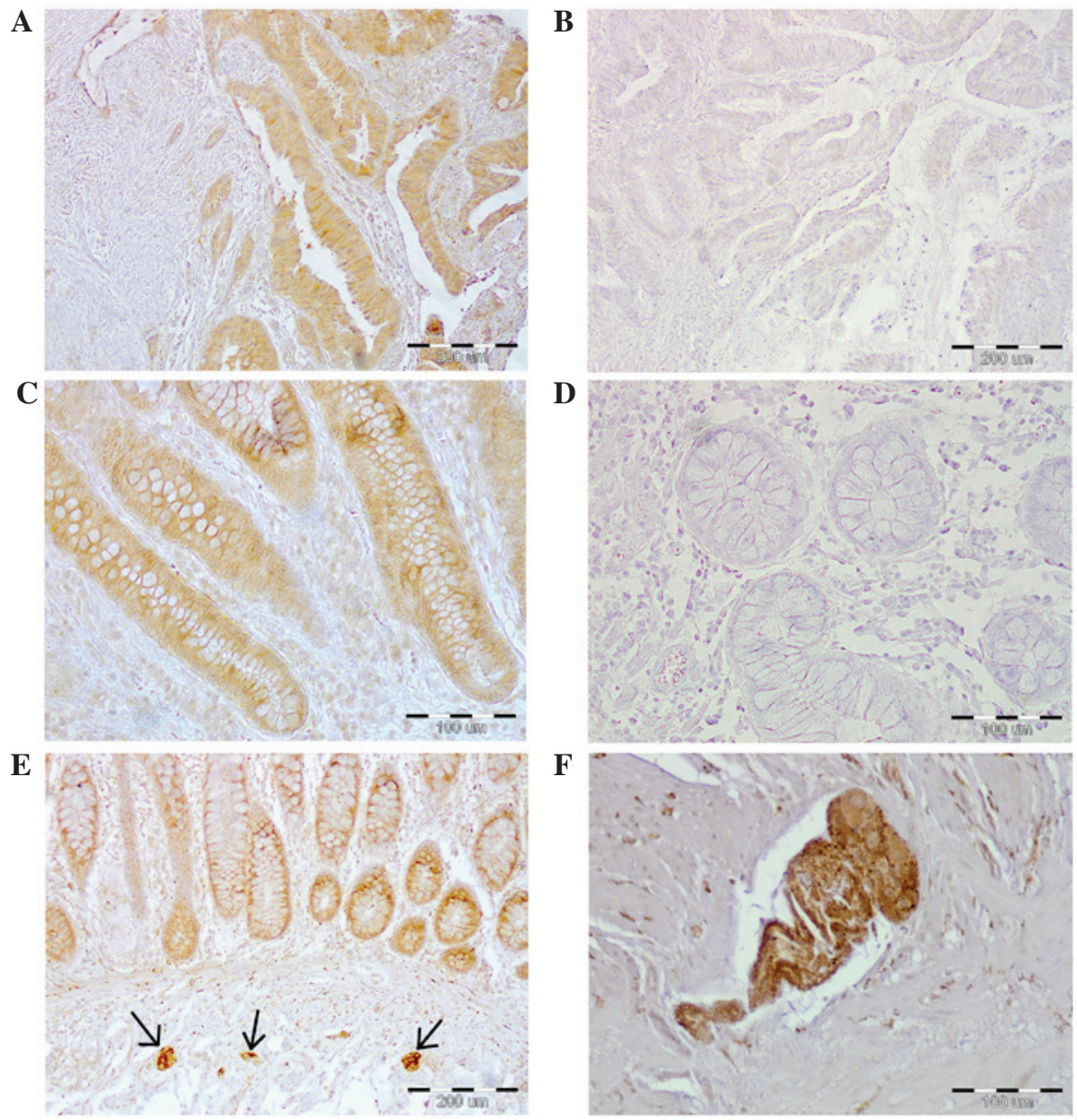

Figure 1. Galanin immunoreactivity in the colon wall of CRC patients. (A) CRC tumor tissue (magnification, x100). (C) Colon mucosa adjacent to the CRC tumor (magnification, x200). (E) Submucosal plexuses (arrows) present in the non-cancerous (distant) section of the colon wall (magnification, x100). Negative controls of (B) CRC tumor (magnification, x100) or (D) mucosa obtained from the non-cancerous (distant) section of the colon wall (magnification, $\mathrm{x} 200$ ). (F) Myenteric plexus from the non-cancerous (distant) section of the colon wall (magnification, x200). CRC, colorectal cancer.

Inc., Burlingame, CA, USA) in PBS for $30 \mathrm{~min}$. The sections were incubated overnight at $4^{\circ} \mathrm{C}$ with rabbit polyclonal anti-human GAL primary antibody (1:400; \#HPA 049864; Sigma-Aldrich; Merck Millipore, Darmstadt, Germany). Sections were then rinsed three times with PBS and incubated with ImmPRESS ${ }^{\text {TM }}$ universal reagent anti-mouse/rabbit immunoglobulin G (commercially diluted; \#MP-7500; Vector Laboratories, Inc.) for $30 \mathrm{~min}$ at room temperature. To visualize the immunoreaction, the sections were immersed in DAB (Dako, Glostrup, Denmark) and counterstained in Mayer's hematoxylin. The slides were dehydrated in ethanol, cleared in xylene and mounted. The specificity of the IHC staining was determined by omitting the primary antibody and replacing it with the same dilution of horse serum. Images of the labeled tissues were captured using a XC-50 camera (Olympus Corporation, Tokyo, Japan) mounted on a light microscope (BX-41; Olympus Corporation). Hematoxylin and eosin staining was performed on separate sections to assess tissue morphology.

Morphometric analysis of the area occupied by myenteric plexuses expressing GAL immunoreactivity. Since the submucosal plexuses occupy a markedly smaller area in the human colon than the myenteric plexuses (20), the latter were selected to assess the area occupied by GAL-Ir structures in the muscularis layer of the colon wall proximal to or distant from the CRC tumor (Fig. 1). In each microscopic field of view (magnification, $\mathrm{x} 4$ ), a large rectangular area with visible GAL-Ir structures and the area of the GAL-Ir myenteric plexuses was determined using Cell^B Imaging Software for Life Science Microscopy version 3.2 (Olympus Corporation). In each patient, the total area occupied by the GAL-Ir neurons in the myenteric plexuses located in the vicinity of and distantly from the tumor was measured in 5-10 fields of view, depending on the size of the section, to encompass the whole length of the border between the circular and longitudinal layers of the muscularis externa. Thus, 22 sections of the large intestinal wall located within close proximity to the tumor and 22 matched sections located distant from the tumor were analyzed to yield the total area of GAL-Ir plexuses, and the ratio of this to the total measured area in a selected histological section of the colon wall was determined (Fig. 2).

Statistical analysis. The concentrations of GAL in the serum of CRC patients and healthy controls and in the tissue homogenates of the patients were analyzed using the Mann-Whitney U-test. The results of morphometric measurement of the area occupied by GAL-Ir myenteric plexuses were analyzed using the Wilcoxon signed-rank test. All statistical analysis was performed using 
Table I. Clinicopathological characteristics of 68 colorectal cancer patients according to the TNM staging system.

\begin{tabular}{lr}
\hline Parameter & $\mathrm{n}(\%)$ \\
\hline Gender & \\
Male & $38(55.9)$ \\
Female & $30(44.1)$ \\
Age, years & \\
$\leq 67$ & $33(48.5)$ \\
$>67$ & $35(51.5)$ \\
Tumor localization & \\
Cecum, ascending and transverse colon & $18(26.5)$ \\
Descending, sigmoid colon, rectum & $50(73.5)$ \\
Depth of invasion (pT status) & \\
T1 & $0(0)$ \\
T2 & $11(16.2)$ \\
T3 & $44(64.7)$ \\
T4 & $13(19.1)$ \\
Lymph node metastases (pN status) & \\
N0 & $33(48.5)$ \\
N1 & $18(26.5)$ \\
N2 & $17(25.0)$ \\
Metastasis (pM status) & \\
M0 & $61(89.7)$ \\
M1 & $7(10.3)$ \\
TNM stage & \\
I & $9(13.2)$ \\
II & $23(33.8)$ \\
III & $29(42.7)$ \\
IV & $7(10.3)$ \\
\hline
\end{tabular}

TNM, Tumor-Node-Metastasis.

Statistica software version 12.5 (StatSoft, Inc., Tulsa, OK, USA). The results were expressed as the mean \pm standard error of the mean (SEM). $\mathrm{P}<0.05$ was considered to indicate a statistically significant difference.

\section{Results}

Patient clinicopathological characteristics. Patient clinicopathological data, including gender, age, tumor location, depth of invasion, lymph node metastasis and TNM stage are presented in Table I.

CRC patients exhibit higher GAL serum concentrations than healthy controls. Analysis of the sera of $68 \mathrm{CRC}$ patients revealed significantly higher mean GAL concentrations than in the 39 healthy volunteers $(119.47 \pm 12.87$ vs. $50.14 \pm 8.13 \mathrm{pg} / \mathrm{ml}$, respectively) (Fig. 3A and B).

Statistical analysis revealed no associations between GAL concentration in the sera of the CRC patients and clinicopathological features, including gender, age, tumor location, depth of invasion, lymph node metastasis and TNM stage (data not shown).
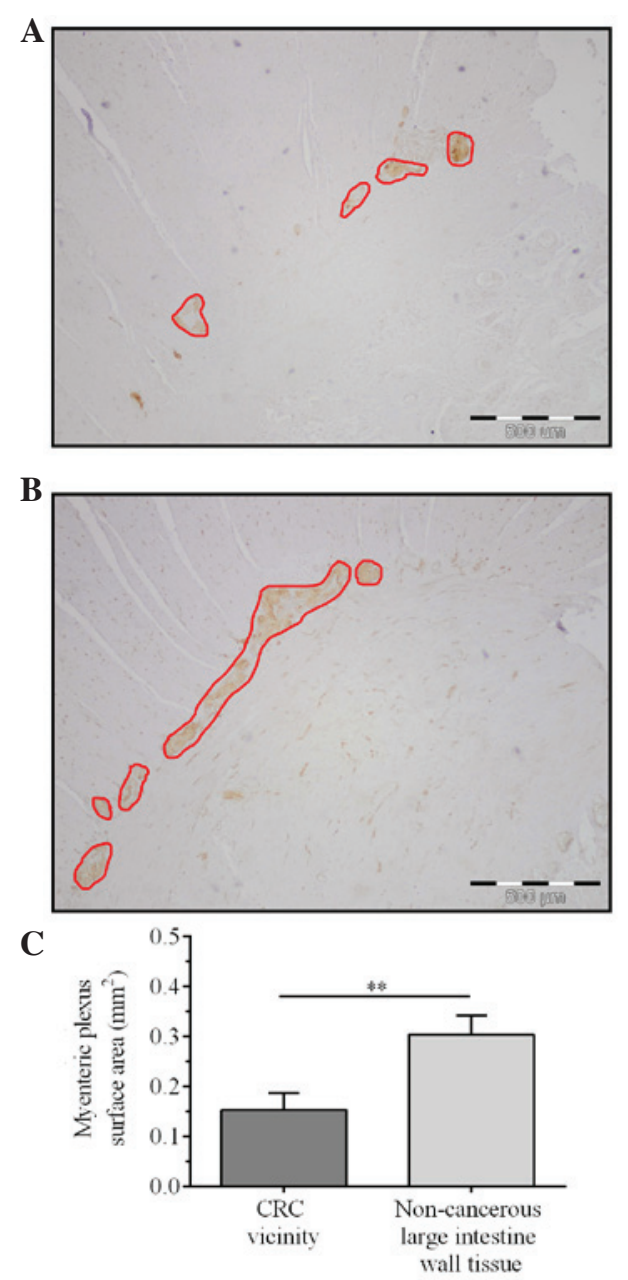

Figure 2. Morphometry of the galanin-immunoreactive myenteric plexuses in the large intestine wall of CRC patients. (A) Plexuses located in the vicinity of the tumor (magnification, x40). (B) Plexuses located distantly from the tumor (magnification, x40). The borders of galanin-immunoreactive myenteric plexuses located between circular and longitudinal muscular layers of the colon wall are outlined by red lines. The black frames outline the constant area of the field of view, which was $\leq 3439,072 \mu \mathrm{m}^{2}$ (artificial empty spaces in certain sections were subtracted). The total area of galanin-immunoreactive myenteric plexuses located close to the tumor was $40,942 \mu \mathrm{m}^{2}$ and the total area of plexuses in the section of non-cancerous colon wall was $112,455 \mu \mathrm{m}^{2}$. (C) Surface area of myenteric plexuses in the vicinity of tumor and in a distant section of non-cancerous colon wall tissue. Bars represent the mean \pm standard error of mean $(n=22)$. ${ }^{* *} \mathrm{P}=0.0071$. CRC, colorectal cancer.

GAL content of CRC tumor tissue homogenates is higher than that of the muscularis externa. The highest levels of GAL were identified in the tumor and mucosa obtained from the non-cancerous section of the intestinal wall (Fig. 4). The mucosal tissues adjacent to the CRC tumor expressed significantly lower levels of GAL $(306.63 \pm 27.60 \mathrm{pg} / \mathrm{g})$ than the tumor tissues $(408.71 \pm 30.28 \mathrm{pg} / \mathrm{g} ; \mathrm{P}=0.019)$ and mucosa from the section of the colon wall distant from the tumor $(462.73 \pm 28.54 \mathrm{pg} / \mathrm{g})$. The GAL concentration of the colon wall muscularis externa obtained adjacent to the tumor $(286.66 \pm 13.43 \mathrm{pg} / \mathrm{g})$ was significantly higher than that of the muscularis distant from the tumor $(175.23 \pm 15.33 \mathrm{pg} / \mathrm{g}$; $\mathrm{P}<0.001)$. The GAL concentration in the CRC tumor was significantly higher than that in either muscularis externa location (Fig. 4; $\mathrm{P}=0.012$ in $\mathrm{CRC}$ vs. $\mathrm{AMM} ; \mathrm{P}<0.001$ in $\mathrm{CRC}$ vs. DMM). 
A

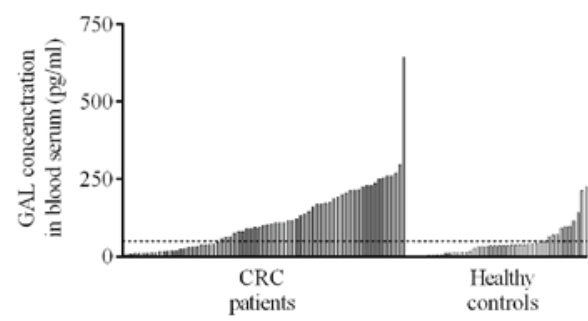

B

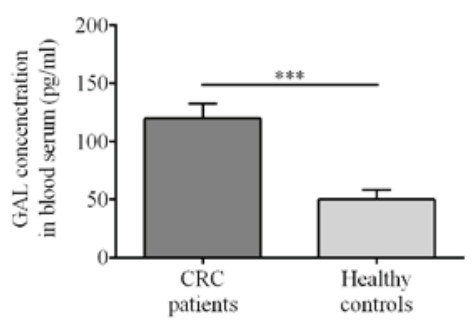

Figure 3. Serum GAL concentration. (A) The variability of GAL serum concentrations in all 68 CRC patients and control subjects. One CRC patient that exhibited a markedly high GAL serum concentration is indicated on the far right. The dashed line denotes the mean GAL concentration in the serum of 39 healthy volunteers. (B) Mean GAL concentration in serum of CRC patients was significantly higher than that of the healthy controls. ${ }^{* * *} \mathrm{P}=0.0002$. GAL, galanin; CRC, colorectal cancer.

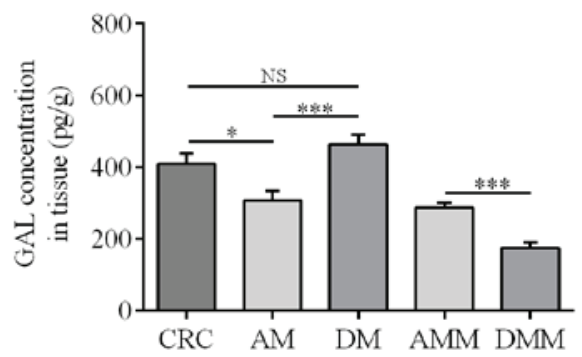

Figure 4. Mean GAL content in the homogenates of tissues obtained from CRC patients. CRC, colorectal cancer; AM, mucosa in the vicinity of the CRC tumor; DM, mucosa located distantly from tumor; AMM, muscularis externa in the vicinity of the CRC tumor; DMM, muscularis externa located distantly from tumor; NS, not significant. ${ }^{*} \mathrm{P}=0.019$, CRC vs. AM; $\mathrm{P}=0.187$, CRC vs. DM (NS); ${ }^{* * *} \mathrm{P}<0.001$, AM vs. DM, and AMM vs. DMM.

CRC tumor and colon wall tissues exhibit strong GAL immunoreactivity in CRC patients. IHC revealed strong GAL immunoreactivity within the cells of the intestinal epithelium, cancer cells and the myenteric plexuses (Fig. 1). The immunoreactivity of GAL in cancer cells, enterocytes and goblet (mucous) cells was predominantly identified in the cytoplasm.

GAL-Ir cells were also observed in the components of the submucosal plexuses and were demonstrated to be smaller in size than the myenteric plexuses from the samples obtained from the colon wall located in the vicinity of and distantly from the tumor (Fig. 1E).

GAL-Ir myenteric plexuses in close proximity to CRC tumors are smaller than those distant from CRC tumors. The examination of the GAL-Ir structures in the myenteric plexuses revealed that the plexuses located in close proximity to the CRC tumor were smaller in size than those located distantly from the cancer (Fig. 2A and B). To assess atrophy, morphometry was used to measure the area occupied by the GAL-Ir myenteric plexuses in the sections of the colon wall of $22 \mathrm{CRC}$ patients.

The mean area of GAL-Ir myenteric plexuses in the intestinal wall proximal to the CRC tumor was $\sim 50 \%$ smaller than that of the distant section of the colon wall $\left(0.15 \pm 0.03 \mathrm{~mm}^{2} \mathrm{vs}\right.$. $0.30 \pm 0.03 \mathrm{~mm}^{2}$, respectively) (Fig. 2).

This finding was confirmed by comparing the relative areas occupied by the GAL-Ir myenteric plexuses in the sections of colon wall close to and distant from the CRC, which were $1.23 \pm 0.85$ and $1.85 \pm 0.56 \%$, respectively. No significant associations were identified between the reduced size of the myenteric plexuses in the vicinity of the tumor and clinicopathological parameters of CRC patients (data not shown).

\section{Discussion}

To the best of our knowledge, this is the first study to demonstrate strong immunoreactivity of GAL in CRC tumors. These morphological findings were validated by the analysis of GAL concentration in neoplastic CRC tissue and two dissected sections of the colon wall: the mucosa and submucosa and the circular and longitudinal layers of muscularis externa with the myenteric plexuses present between them. Additionally, using morphometry, the atrophy of myenteric plexuses was identified in the vicinity of CRC tumors. Thus, we hypothesize that the elevated concentration of GAL in the serum of CRC patients may be caused by the secretion of this neuropeptide by cancer cells, however, the possibility that other cell types or tissues may be involved cannot be excluded.

The present study is the first to report that CRC patients exhibit a statistically significant higher serum concentration of GAL than healthy patients, as the marked increase in serum GAL levels reported previously in 66 CRC Korean patients was not statistically significant (14). Kim et al (14) postulated that the CRC tumor was the source of the increased serum GAL concentration, as the levels of GAL mRNA were markedly increased in the CRC tumor; however, the concentration of the neuropeptide, GAL, was not measured (14). Similarly, Nagayoshi et al (21) reported that GAL mRNA levels in 112 tumor samples obtained from CRC patients were significantly higher than that in 27 normal colon mucosa samples; however, the concentration of GAL was not determined.

In the present study, GAL concentration was measured in both the homogenates of the CRC tumor and two major sections of the colon wall by ELISA assay, which provided notable results. High GAL levels were observed not only in the CRC tumor, but also in the homogenates of mucosa and submucosa from the non-cancerous section of the colon wall. The GAL concentration of the tumor was significantly higher than that in the homogenates of the muscularis layer of the colon wall, which are known to contain GAL in the myenteric plexuses $(6,17)$, and intense GAL immunoreactivity was 
observed in the cytoplasm of CRC tumor cells, which indicates that the CRC tumor is an important source of GAL in the sera of CRC patients. GAL immunoreactivity has also been observed in the cells of neuroendocrine tumors, including neuroblastomas (11), pituitary adenomas (10) and paragangliomas (22), as well as in a variety of non-neuroendocrine tumors, such as melanomas (13), glioblastomas (23) and embryonic carcinomas (24).

Furthermore, in the present study IHC demonstrated, for the first time, the presence of GAL-like immunoreactivity in the epithelium of human colon mucosa, which had only been demonstrated in human submucosal (17) and myenteric nervous plexuses to date $(6,17)$. The presence of cytoplasmic GAL immunoreactivity in the neoplastic cells of CRC tumors and in the epithelial cells of the mucosa of the large intestine may be associated with the function of GAL as a regulator of epithelial secretion and proliferation $(25,26)$. This hypothesis is supported by the fact that GAL has been identified in the human epidermis and the ductal cells of sweat glands (27), which are epithelial tissues of different embryonic origin than the intestine.

The presence of cytoplasmic GAL immunoreactivity in neoplastic CRC cells may be associated with the function of GAL as a regulator of cellular proliferation under pathological conditions. In the model HCT116 CRC cell line, silencing of the GAL receptor 1 (GaLR1) or silencing of GAL, induces caspase 8-dependent apoptosis, which suggests that GaLR1/GAL may present a potential drug target for CRC chemotherapy (28). Additionally, in pancreatic cancer cell lines (29), small-cell lung cancer (30) and rat pituitary tumors (31), GAL interacts with GaLR1 to activate the mitogen-activated protein kinase signaling pathway and induces mitogenic activity of these cancer cells. However, it has been also suggested that GAL may exhibit a protective function in head and neck squamous cell cancer (HNSCC), since GaLR1 and 2 inhibit the proliferation of HNSCC cells via extracellular signal-regulated kinase 1/2-mediated effects on cell-cycle control proteins (32).

The morphometric analysis in the present study provided a quantitative aspect to the observations of Godlewski (18), who reported alterations in the structure of the ENS components in the vicinity of the CRC tumor. Notably, in the present study, the muscularis externa in the vicinity of the CRC invasion contained higher GAL levels than that in the distant section, which indicates the increased synthesis of GAL or the release of this neuropeptide from the atrophic myenteric plexuses into the surrounding tissue. Thus, this indicates that the myenteric plexuses located close to the CRC tumor undergo morphological and thus, functional alterations that may also affect the function of the colon wall.

In conclusion, the present study comprehensively investigated the presence of GAL in the colon wall of CRC patients. To the best of our knowledge, this study is the first to demonstrate GAL immunoreactivity in the epithelium of the human colon and CRC tumor cells, as well as high GAL levels in CRC tumors. In addition, increased GAL levels were identified in the muscularis externa, despite the atrophy of the myenteric plexuses in the vicinity of the CRC tumor, when compared with the muscularis located distantly from the tumor. The results indicate that serum and tissue GAL levels may present useful potential biomarkers in CRC patients. However, the broad range of GAL serum levels identified in both CRC patients and controls and reported by Kim et al (14) indicate that further studies using larger patient cohorts are required to establish the clinical significance of these findings.

\section{Acknowledgments}

This study was supported by The University of Warmia and Mazury (Olsztyn, Poland; grant no. 1507-0881). The authors would like to thank Dr. Ewa Wędrowska for performing the statistical analysis for the study.

\section{References}

1. Ferlay J, Soerjomataram I, Ervik M, Dikshit R, Eser S, Mathers C, Rebelo M, Parkin DM, Forman D and Bray F: GLOBOCAN 2012 v1.0, Cancer Incidence and Mortality Worldwide: IARC CancerBase No. 11 [Internet]. Lyon, France: International Agency for Research on Cancer; 2013. http://globocan.iarc.fr. Accessed January 16, 2015.

2. Keane MG and Johnson GJ: Early diagnosis improves survival in colorectal cancer. Practitioner 256: 15-18,2012.

3. Chen H, Zucknick M, Werner S, Knebel P and Brenner H: Head-to-head comparison and evaluation of 92 plasma protein biomarkers for early detection of colorectal cancer in a true screening setting. Clin Cancer Res 21: 3318-3326, 2015.

4. Gonzalez-Pons $M$ and Cruz-Correa M: Colorectal cancer biomarkers: Where are we now? Biomed Res Int 2015: 149014, 2015.

5. Mitsukawa K, Lu X and Bartfai T: Galanin, galanin receptorss, and drug targets. In: Galanin. Hökfelt T (ed). Vol. 102. Springer, Basel, pp-pp7-9, 2010.

6. Burleigh DE and Furness JB: Distribution and actions of galanin and vasoactive intestinal peptide in the human colon. Neuropeptides 16: 77-82, 1990.

7. von Rosenvinge EC and Raufman JP: Gastrointestinal peptides and regulation of gastric acid secretion. Curr Opin Endocrinol Diabetes Obes 17: 40-43, 2010.

8. Barreto SG, Carati CJ, Toouli J and Saccone GT: The islet-acinar axis of the pancreas: More than just insulin. Am J Physiol Gastrointest Liver Physiol 299: G10-G22, 2010.

9. Bauer FE, Hacker GW, Terenghi G, Adrian TE, Polak JM and Bloom SR: Localization and molecular forms of galanin in human adrenals: Elevated levels in pheochromocytomas. J Clin Endocrinol Metab 63: 1372-1378, 1986.

10. Grenbäck E, Bjellerup P, Wallerman E, Lundblad L, Anggård A, Ericson K, Aman K, Landry M, Schmidt WE, Hökfelt T and Hulting AL: Galanin in pituitary adenomas. Regul Pept 117: $127-139,2004$.

11. Tuechler C, Hametner R, Jones N, Jones R, Iismaa TP, Sperl W and Kofler B: Galanin and galanin receptor expression in neuroblastoma. Ann NY Acad Sci 863: 438-441, 1998.

12. Sugimoto T, Seki N, Shimizu S, Kikkawa N, Tsukada J, Shimada H, Sasaki K, Hanazawa T, Okamoto Y and Hata A: The galanin signaling cascade is a candidate pathway regulating oncogenesis in human squamous cell carcinoma. Genes Chromosomes Cancer 48: 132-142, 2009.

13. Gilaberte Y, Vera J, Coscojuela C, Roca MJ, Parrado C and González S: Expression of galanin in melanocytic tumors. Actas Dermosifiliogr 98: 24-34, 2007 (In Spanish).

14. Kim KY, Kee MK, Chong SA and Nam MJ: Galanin is up-regulated in colon adenocarcinoma. Cancer Epidemiol Biomarkers Prev 16: 2373-2378, 2007.

15. Ries LA, Wingo PA, Miller DS, Howe HL, Weir HK, Rosenberg HM, Vernon SW, Cronin K and Edwards BK: The annual report to the nation on the status of cancer, 1973-1997, with a special section on colorectal cancer. Cancer 88: 2398$2424,2000$.

16. Godlewski J and Łakomy IM: Changes in vasoactive intestinal peptide, pituitary adenylate cyclase- activating polypeptide and neuropeptide Y-ergic structures of the enteric nervous system in the carcinoma of the human large intestine. Folia Histochem Cytobiol 48: 208-216, 2010. 
17. Godlewski J and Pidsudko Z: Characteristic of galaninergic components of the enteric nervous system in the cancer invasion of human large intestine. Ann Anat 194: 368-372, 2012.

18. Godlewski J: Morphological changes in the enteric nervous system caused by carcinoma of the human large intestine. Folia Histochem Cytobiol 48: 157-162, 2010.

19. Godlewski J, Krazinski BE, Kiezun J, Kwiatkowski P, Sulik M, Tenderenda M, Biernat W and Kmiec Z: PLAGL1 protein is differentially expressed in the nephron segments and collecting ducts in human kidney. Folia Histochem Cytobiol 53: 96-104, 2015.

20. Furness JB (ed): Structure of the enteric nervous system. In: The Enteric Nervous System. Wiley-Blackwell, Hoboken, NJ, p6, 2006.

21. Nagayoshi K, Ueki T, Tashiro K, Mizuuchi Y, Manabe T, Araki H, Oda Y, Kuhara S and Tanaka M: Galanin plays an important role in cancer invasiveness and is associated with poor prognosis in stage II colorectal cancer. Oncol Rep 33: 539-546, 2015.

22. Tadros TS, Strauss RM, Cohen C and Gal AA: Galanin immunoreactivity in paragangliomas but not in carcinoid tumors. Appl Immunohistochem Mol Morphol 11: 250-252, 2003

23. Berger A, Santic R, Almer D, Hauser-Kronberger C, Huemer M, Humpel C, Stockhammer G, Sperl W and Kofler B: Galanin and galanin receptors in human gliomas. Acta Neuropathol 105 : 555-560, 2003.

24. Skotheim RI, Lind GE, Monni O, Nesland JM, Abeler VM Fosså SD, Duale N, Brunborg G, Kallioniemi O, Andrews PW and Lothe RA: Differentiation of human embryonal carcinomas in vitro and in vivo reveals expression profiles relevant to normal development. Cancer Res 65: 5588-5598, 2005.

25. Benya RV, Marrero JA, Ostrovskiy DA, Koutsouris A and Hecht G: Human colonic epithelial cells express galanin-1 receptors, which when activated cause $\mathrm{Cl}$ - secretion. Am J Physiol 276: G64-G72, 1999.
26. Lang R, Gundlach AL, Holmes FE, Hobson SA, Wynick D, Hökfelt T and Kofler B: Physiology, signaling and pharmacology of galanin peptides and receptors: Three decades of emerging diversity. Pharmacol Rev 67: 118-175, 2015.

27. Kofler B, Berger A, Santic R, Moritz K, Almer D, Tuechler C, Lang R, Emberger M, Klausegger A, Sperl W and Bauer JW: Expression of neuropeptide galanin and galanin receptors in human skin. J Invest Dermatol 122: 1050-1053, 2004.

28. Stevenson L, Allen WL, Turkington R, Jithesh PV, Proutski I, Stewart G, Lenz HJ, Van Schaeybroeck S, Longley DB and Johnston PG: Identification of galanin and its receptor GalR1 as novel determinants of resistance to chemotherapy and potential biomarkers in colorectal cancer. Clin Cancer Res 18: 5412-5426, 2012.

29. Tjomsland V and El-Salhy M: Effects of single, double or triple combinations of octreotide, galanin and serotonin on a human pancreatic cancer cell line. Histol Histopathol 20: 537-541, 2005.

30. Seufferlein T and Rozengurt E: Galanin, neurotensin, and phorbol esters rapidly stimulate activation of mitogen-activated protein kinase in small cell lung cancer cells. Cancer Res 56: 5758-5764, 1996

31. Hammond PJ, Smith DM, Akinsanya KO, Mufti WA, Wynick D and Bloom SR: Signaling pathways mediating secretory and mitogenic responses to galanin and pituitary adenylate cyclase-activating polypeptide in the 235-1 clonal rat lactotroph cell line. J Neuroendocrinol 8: 457-464, 1996.

32. Kanazawa T, Misawa K, Misawa Y, Uehara T, Fukushima H, Kusaka G, Maruta M and Carey TE: G-protein-coupled receptors: Next generation therapeutic targets in head and neck cancer? Toxins (Basel) 7: 2959-2984, 2015. 